\title{
ANÁLISE DAS INTERVENÇÕES FARMACÊUTICAS REALIZADAS EM UMA UNIDADE BÁSICA DE SAÚDE DO MUNICÍPIO DE CRICIÚMA
}

DOI: http://dx.doi.org/10.18616/prat01

\author{
Janaína dos Santos Nunes \\ UNESC - Universidade do Extremo Sul Catarinense \\ jana.snunes@hotmail.com \\ Jóyce Pereira Ricardo \\ UNESC - Universidade do Extremo Sul Catarinense \\ joycericardo@hotmail.com \\ Marilia Schutz Borges \\ UNESC - Universidade do Extremo Sul Catarinense \\ msb@unesc.net
}




\section{INTRODUÇÃO}

A Atenção Básica é o primeiro nível de acesso dos usuários ao sistema de saúde, abrangendo cuidados essenciais aos principais problemas da comunidade (GIOVANELLA; MENDONLA, 2012). De acordo com os princípios que orientam o Sistema Único de Saúde (SUS), a Atenção Básica tem como estratégia firmar a reorientação da política de saúde no país, exercendo atividade de interligação e aprimoramento do eixo ordenador de toda a rede de atenção à saúde. É essencial que a atenção básica caminhe pelos princípios da universalidade, acessibilidade, integralidade, humanização, equidade e da participação social (ESCOREL et al., 2007; TANAKA, 2011; MAEYAMA et al., 2018).

No âmbito da atenção básica, contamos com a inserção do farmacêutico atuando na equipe multidisciplinar nas Unidades Básicas de Saúde (UBSs), de forma a realizar ações de cuidado à saúde dos usuários. As UBSs desempenham papel central na garantia de acesso da população a uma atenção à saúde de qualidade (MELO; CASTRO, 2017).

Neste contexto, é importante destacar a existência da Política Nacional de Medicamentos, que realiza ações para promover melhorias de assistência à saúde da população, visando assim a eficácia, a garantia da segurança, e a qualidade dos medicamentos fornecidos (BRASIL, 1998; VASCONCELOS et al., 2017). Diante disto, a assistência farmacêutica representa ações interligadas à promoção, proteção e recuperação da saúde, tanto no âmbito individual como no âmbito coletivo (MARIM et al., 2003; LEITE et al., 2016).

Entre as áreas da assistência farmacêutica existe o cuidado farmacêutico, que tem como objetivo beneficiar o paciente com a terapia segura e efetiva, atender às necessidades farmacoterapêuticas (BERNARDI et al., 2014), prevenir agravos, promover educação em saúde e o uso racional de medicamentos (MINISTÉRIO DA SAÚDE, 2014).

A promoção do uso racional de medicamentos tem como finalidade orientar o uso dos medicamentos, por meio de estratégias e atividades que visam promover a melhor escolha de acordo com evidências científicas, com- 
provando sua segurança e efetividade (BRASIL, 2013a). Além disso, proporciona ao paciente uma dispensação adequada com as orientações devidamente explicadas (WHO, 1985; AQUINO, 2008; OMS, 2015; PINTO, 2015).

Uma das áreas que vai ao encontro destas ações é a farmácia clínica. Nessa atuação, o profissional presta zelo ao paciente, de forma a aperfeiçoar a farmacoterapia, possibilitando saúde, bem-estar e a prevenção de agravos. (BRASIL, 2013b). Esta prática, segundo a resolução n. 586/2013, pode ser realizada através de consultas, onde o farmacêutico faz a anamnese e seleciona terapias farmacológicas e não farmacológicas. Além disso, é possível fazer outras intervenções, inclusive encaminhamentos para os demais profissionais da saúde (ROZENFELD; FONSECA; ACURCIO, 2008; BRASIL, 2013c; MARTIN; FABER, 2016; COSTA et al., 2017).

Os serviços clínicos são importantes; pois, segundo dados do Ministério da Saúde, no mundo, mais de 50\% dos medicamentos são prescritos, dispensados ou vendidos de forma inadequada. Além disso, mais de $50 \%$ dos pacientes utilizam fármacos incorretamente, aumentando os índices de morbidade e mortalidade (MINISTÉRIO DA SAÚDE, 2012).

$\mathrm{O}$ uso inadequado de medicamentos também está relacionado à polimedicação, automedicação e na prescrição em desacordo com as diretrizes clínicas (MARIN et al., 2008). Os medicamentos, quando consumidos em doses acima do recomendado, ou até mesmo os não prescritos, podem gerar consequências no organismo, desde uma reação alérgica até mesmo o óbito (MINISTÉRIO DA SAÚDE, 2015). Dados alarmantes publicados pelo Sistema Nacional de Informações Tóxico-Farmacológicos (SINITOX) demonstram que os medicamentos ocupam a primeira posição dos agentes intoxicantes em seres humanos desde 1999 e, no ano de 2016, foram responsáveis por 40,10\% dos casos registrados por intoxicação (SINITOX, 2016).

O farmacêutico é o profissional que pode colaborar para a redução destes problemas, como foi observado no estudo realizado no Hospital das Clínicas da Faculdade de Medicina da Universidade de São Paulo. Os autores avaliaram 386 prescrições de pacientes e, após análise, foram propostas 212 intervenções farmacêuticas, sendo que destas, $64,3 \%$ foram aceitas pelos pres- 
critores. As intervenções envolviam indicações farmacoterapêuticas, orientações para ajustes de dose, redução do uso de medicamentos potencialmente inapropriados, ajustes de prescrição, entre outras. Este dado reforça o papel do farmacêutico, quanto ao cuidado do paciente juntamente com a equipe multidisciplinar (VIANA; ARANTES; RIBEIRO, 2017).

Tendo em vista o tema abortado, o objetivo deste trabalho foi avaliar o desfecho das intervenções farmacêuticas na prestação ao cuidado do paciente em uma UBS do município de Criciúma.

\section{MÉTODOS}

O presente trabalho trata-se de uma avaliação descritiva transversal de caráter quantitativo. A pesquisa foi realizada em uma UBS do município de Criciúma-SC. O público-alvo são pacientes maiores de 18 anos que receberam intervenções da farmacêutica na UBS no período de outubro de 2017 a maio de 2018. Esta análise contou também com os dados dos prontuários dos pacientes no sistema informatizado, onde constava a evolução dos casos.

Foi realizado contato telefônico com os pacientes para agendar um horário na UBS, no mês de agosto de 2018. A coleta de dados foi realizada através de um questionário com perguntas abertas e fechadas, contendo questões sobre o uso racional de medicamentos, as intervenções farmacêuticas realizadas, aspectos sociodemográficos e a concepção dos pacientes em relação à importância de ter o profissional farmacêutico presente na UBS.

Os participantes receberam uma breve explicação sobre os objetivos do trabalho, onde foi entregue o Termo de Consentimento Livre e Esclarecido, que foi assinado pelos participantes. Após terem aceitado, foram convidados a responder o questionário de forma voluntária, anônima, não remunerada $\mathrm{e}$ com fins científicos.

Após a coleta das informações, os dados foram analisados pelo programa Microsoft Office Excel e apresentados em forma de gráficos, tabelas, texto corrido ou porcentagem de acordo com as características dos dados coletados. 
A pesquisa foi realizada mediante o consentimento da secretária de Saúde do município de Criciúma.

Esta pesquisa foi aprovada pelo Comitê de Ética em Pesquisa e Humanos (CEP) da Universidade do Extremo Sul Catarinense, sob parecer de número 2.744.889.

\section{RESULTADOS}

De um total de 102 pessoas que receberam intervenções da farmacêutica na UBS, 60 pacientes participaram deste estudo. Em relação aos demais, o contato não foi possível devido a diversos motivos; entre eles, pode-se destacar: falecimento, pacientes menores de 18 anos, mudança de cidade, não atenderam a ligação ou não quiserem participar da pesquisa.

A partir da pesquisa concluída com os 60 pacientes, verificou-se que $65 \%(n=39)$ eram do sexo feminino e $35 \%(n=21)$ do sexo masculino $35 \%$ $(n=21)$.

A tabela 1 representa a distribuição dos pacientes de acordo com sua idade. Para melhor ilustração e análise, esse parâmetro foi agrupado por intervalos de classe. Verificou-se que a faixa etária predominante foi de 71 a 80 anos, que foram a grande maioria entre os estudados, com $40 \%(\mathrm{n}=24)$.

Tabela 1 - Idade dos pacientes que receberam intervenções da farmacêutica da UBS de Criciúma.

\begin{tabular}{c|c|c}
\hline Faixa etária & Quantidade & Porcentagem \\
\hline 31 a 40 & 2 & $3,33 \%$ \\
\hline 51 a 60 & 13 & $21,67 \%$ \\
\hline 61 a 70 & 19 & $31,67 \%$ \\
\hline 71 a 80 & 24 & $40,00 \%$ \\
\hline 81 a 90 & 2 & $3,33 \%$ \\
\hline Total geral & $\mathbf{6 0}$ & $\mathbf{1 0 0 , 0 0 \%}$ \\
\hline
\end{tabular}

Fonte: Autores, 2018 


\section{PRÁTICAS E SABERES EM

Em relação ao grau de escolaridade, verificou-se que o ensino fundamental completo e incompleto obtiveram a mesma porcentagem $(38,33 \%)$, conforme mostra a tabela 2 .

Tabela 2 - Escolaridade dos pacientes que receberam intervenções da farmacêutica na UBS de Criciúma.

\begin{tabular}{c|c|c}
\hline Escolaridade & Quantidade & Porcentagem \\
\hline Analfabeto & 1 & $1,67 \%$ \\
\hline Ens. fund. Incompleto & 23 & $38,33 \%$ \\
\hline Ens. fund. Completo & 23 & $38,33 \%$ \\
\hline Ens. médio incompleto & 5 & $8,33 \%$ \\
\hline Ens. médio completo & 6 & $10,00 \%$ \\
\hline Ens. sup. Incompleto & 1 & $1,67 \%$ \\
\hline Ens. sup. Completo & 1 & $1,67 \%$ \\
\hline Total geral & $\mathbf{6 0}$ & $\mathbf{1 0 0 , 0 0 \%}$ \\
\hline
\end{tabular}

Fonte: Autores, 2018.

Gráfico 1 - Intervenções que os pacientes receberam da farmacêutica da UBS de Criciúma.

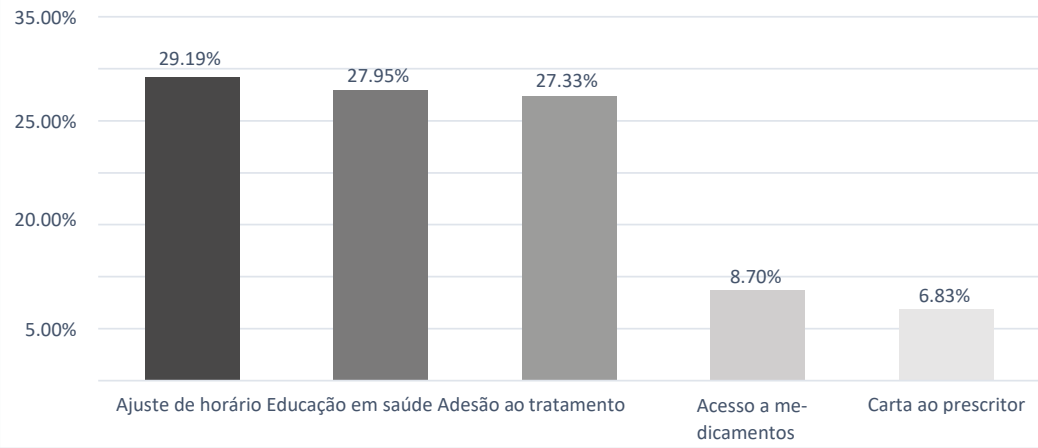

Fonte: Autores, 2018

Quanto aos pacientes que receberam a carta elaborada pela farmacêutica, todos $(n=11)$ entregaram-na para o prescritor, para que assim o mesmo pudesse analisar melhor o quadro de saúde. Quanto a aceitação da 


\section{PRÁTICAS E SABERES EM

conduta farmacêutica por parte dos prescritores, 72,72\% $(n=8)$ acataram o parecer farmacêutico, conforme o relato dos pacientes.

No que diz respeito a dúvidas com medicamentos, 78,33\% $(\mathrm{n}=47)$ dos entrevistados relataram saná-las com a farmacêutica. Quanto aos horários de administração dos medicamentos, $90 \%(n=54)$ dos pacientes relataram que a farmacêutica orienta sobre a posologia correta e, apenas $10 \%(n=6)$, relataram que não receberam orientação.

Sobre as orientações da farmacêutica quanto ao controle das doenças dos pacientes, $78,33 \%(n=47)$ responderam que recebem orientações. Dentre estas doenças, pode-se destacar: diabetes mellitus, hipertensão, hipercolesterolemia, insuficiência cardíaca congestiva, osteoporose, hipotireoidismo, entre outras.

No tocante à importância da farmacêutica no atendimento prestado aos pacientes, o gráfico 2 ilustra a classificação realizada, tais como: ótimo, bom, regular e ruim. Verificou-se um percentual de 60\% $(n=36)$ com nível ótimo e $31,67 \%(n=19)$ com nível bom.

Gráfico 2 - A importância da farmacêutica no atendimento prestado aos pacientes da UBS de Criciúma.

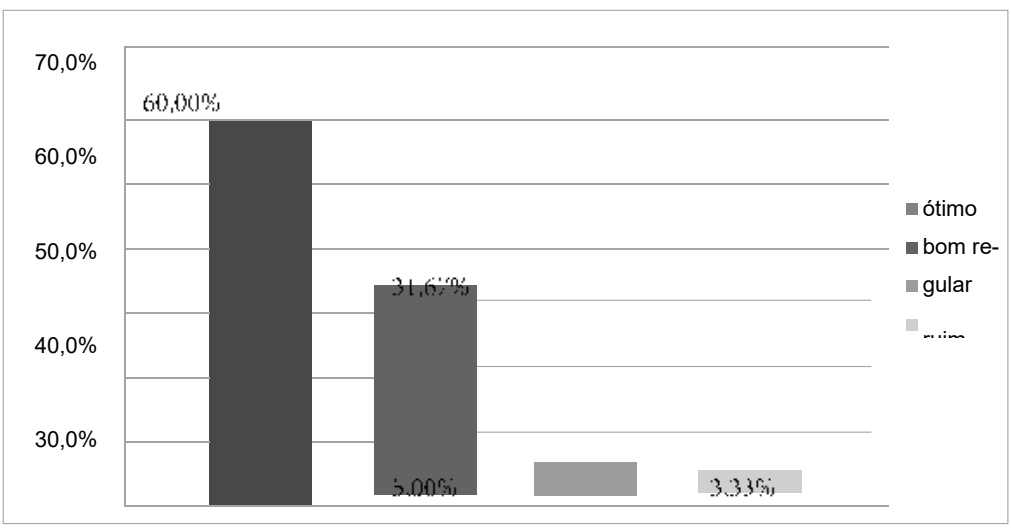

Fonte: Autores, 2018 
A maioria das justificativas das respostas foram positivas, totalizando $90 \%(\mathrm{n}=54)$, como pode ser observado na fala dos pacientes, que relataram: "Foi importante para entender o meu tratamento, a gente lê a bula do remédio e não entende nada, então acho importante". Um outro paciente relatou: "porque faz uma boa orientação do medicamento, ajusta os medicamentos no horário certo, ajuda muito". E apenas 3,33\% ( $\mathrm{n}=2)$ justificaram com pontos negativos, como percebe-se na fala de um dos pacientes: "não sei nem se quem me atende é a farmacêutica" e, 6,66\% (n=4) não quiseram justificar.

Foi questionado sobre a orientação fornecida pela profissional farmacêutica e se a mesma contribui para a adesão do tratamento. A maioria dos pacientes, representados por $91,67 \%(n=55)$, responderam que a mesma contribui. Quando questionado o porquê, 95\% $(n=57)$ das respostas foram positivas, como observado na fala de um dos pacientes, que disse: "Muito importante, porque não entendemos de medicamentos, e ela entrega uma folha explicando como tomar os medicamentos, e nos esclarece. Tudo beleza”. Já outro paciente relatou: "Porque ela explica bem, orienta bem, e assim tomo o medicamento correto".

Outros relatos semelhantes aos descritos acima também foram citados pelos pacientes. São justificativas positivas que demonstram a importância do papel da farmacêutica para um melhor resultado no tratamento, agindo sempre com segurança, paciência, conhecimentos técnicos importantes, garantindo à população, cuidado e saúde de qualidade.

\section{DISCUSSÃO}

O dado alcançado quanto ao parâmetro de sexo está em concordância com a literatura, onde evidencia as mulheres como maioria na busca pelos serviços de saúde. Atualmente as instituições mostram ações pouco valorizadas pelos homens, principalmente os serviços de promoção e proteção à saúde (GONÇALVES; FARIA, 2016; OLIVEIRA et al., 2016; LEVORATO, 2014). Esse resultado pode ser atribuído ao fato de que no sexo feminino, existe priorização das políticas de atenção voltadas à saúde da mulher e, que 
em geral, a mulher necessita realizar exames periódicos ginecológicos, e assim utilizando com maior frequência os serviços de saúde. Já o sexo masculino, no que se trata de cuidados coma saúde, tem-se a visão de senso comum que o homem é um ser forte, que dificilmente adoece, buscando assim os serviços de saúde em casos extremos, quando a doença já está alojada (TONELI; SOUZA; MULLER, 2010).

O presente estudo verificou que $75 \%$ dos pacientes que participaram da pesquisa são classificados como idosos, e segundo o Ministério da Saúde, o idoso é definido como a pessoa que tem 60 anos ou mais de idade (MINISTÉRIO DA SAÚDE, 2010), visto que o envelhecimento é um processo dinâmico e progressivo que engloba mudanças orgânicas, funcionais e psicossociais em um indivíduo (GOIS; PERNAMBUCO; LIMA, 2018; DAWALIBI et al., 2013). No Brasil, o aumento da população idosa vem ocorrendo de forma rápida e progressiva, entre os homens, a expectativa de vida aos 60 anos aumentou para 75,6 anos e, entre as mulheres foram ainda maiores, aumentou para 82,5 anos (ANDRADE et al., 2013).

Já em relação à escolaridade, um estudo analisou este fator ao entendimento das patologias, onde foi destacado que a escolaridade pode interferir na obtenção das informações sobre determinados conteúdos, o que pode ser uma característica repetida na população em que estes são acolhidos nos serviços de saúde pública (RODRIGUES et al., 2012; MACHADO; ALMEIDA, 2017).

Em contrapartida, um estudo realizado na cidade de São Paulo onde a variação de escolaridade foi de 1 a 17 anos, mostrou que apenas 32,4\% apresentaram letramento inadequado (GOULART et al., 2009). Assim sendo, não se trata apenas da capacidade do indivíduo de saber ler e escrever, mas sim sua capacidade para com tais habilidades, especificamente no âmbito da saúde (SAMPAIO et al., 2015), visto que, letramento em saúde é a capacidade que o indivíduo apresenta em compreender informações e instruções, com o intuito de escolher decisões adequadas para sua saúde (MARAGNO; LUIZ, 2016; WEISS et al., 2005). 
No que diz respeito ao atendimento recebido pela farmacêutica, a grande maioria afirmou ter recebido orientações. Este resultado está em conformidade com a literatura, que evidencia o farmacêutico como um profissional que instrui sobre utilização e dosagens corretas, possíveis interações medicamentosas, reações adversas, perigos na automedicação entre outras orientações (ALVES et al., 2017; ANDRADE; SILVA; FREITAS, 2005).

Um estudo realizado com pacientes portadores da síndrome metabólica mostrou que as intervenções farmacêuticas no ambiente domiciliar foram efetivas, ocorrendo a diminuição de 59\% de problemas relacionados a medicamentos, e houve o aumento de $18,2 \%$ da adesão medicamentosa. Este dado evidencia a importância do cuidado integral a cada paciente com a conscientização de mudanças no estilo de vida e no tratamento farmacológico (AZEVEDO et al., 2017).

O percentual de aceitabilidade por parte dos prescritores das intervenções farmacêuticas foi de $88 \%$, resultado compatível a outro estudo realizado em uma UBS de São Paulo entre os anos de 2010 e 2011, no qual o prescritor apresentou concordância com o farmacêutico em $67,8 \%$ para realizar modificações da farmacoterapia dos pacientes (MELO et al., 2017).

Em outro estudo, as intervenções mostram que a consulta com o farmacêutico é uma forma de introduzir o paciente na atenção primária, já que as consultas são desenvolvidas para que o paciente busque atender suas necessidades como um todo. Os encaminhamentos para os demais profissionais, sejam eles por carta ou pessoalmente, reforça o trabalho centrado no paciente nas unidades de saúde (LOMBARDI, 2016). Além disso, segundo Curran et al. (2018), o diálogo entre os médicos da atenção primária e os farmacêuticos comunitários é de grande importância pois aperfeiçoa o compartilhamento de informações e a boa tomada de decisões.

Referindo-se a dúvidas com medicamentos, outros estudos realizados demonstram que o farmacêutico é considerado um dos profissionais de saúde mais compreensível, tendo o papel de aconselhador do paciente. Além disso, auxilia na farmacoterapia e também com outras alternativas não farmacológicas (CAVACO et al., 2018; YEGENOGLU; BAYDAR, 2011). 
A orientação do farmacêutico sobre os horários da administração dos medicamentos é uma informação que faz a diferença para o sucesso da farmacoterapia. Segundo Schenkel et al. (2012), por intermédio da farmacocinética e da farmacodinâmica, pode-se definir a posologia mais apropriada para cada medicamento, sendo que o intervalo entre as doses é essencial para manter a efetividade do tratamento. Consequentemente a dose apropriada não deve ser restringida e nem acrescentada, sob pena de não causar concentrações com deficiência no tratamento ou provocar toxicidade no organismo de cada paciente.

A orientação sobre doenças crônicas também foi evidenciada no presente estudo. De acordo com Alves et al. (2017), o farmacêutico possui a competência de informar sobre medicamentos prescritos e quando retirados no ato da dispensação, pois o profissional orienta sobre problemas de saúde e as doenças crônicas, possibilitando uma melhor adesão ao tratamento.

Dentre essas doenças crônicas que o farmacêutico pode estar realizando orientações, destaca-se as não transmissíveis, que são: doenças cardiovasculares (como a hipertensão e hipercolesterolemia), diabetes, doenças respiratórias crônicas e câncer (SCHMIDT et al., 2011; MANSO; GERARDI, 2015; SCHWEITZER et al., 2018). Estas patologias estão em concordância com a maioria das doenças citadas no presente estudo. Podemos citar também que, no Sul do Brasil, as doenças que apresentam maior prevalência são as cardiovasculares (SCHMIDT et al., 2011). Outro estudo mostrou que as doenças crônicas não transmissíveis se apresentaram como sendo o maior problema de saúde do Brasil (MALTA et al., 2017a) e a mortalidade em 2015 aumentou para 75,8\% (MALTA et al., 2017b).

A profissão farmacêutica evoluiu muito nos últimos tempos na maioria dos países, oferecendo uma abordagem mais acessível e clara para auxílio no alcance do tratamento. Atualmente está ocorrendo mudanças positivas, diversificando o papel do farmacêutico, indo mais além do que apenas o ato de dispensar medicamentos (WIDAYATI et al., 2017). Assim, podemos confirmar que a presente pesquisa está em conformidade com a literatura, uma vez que $91,67 \%$ dos pacientes responderam que a farmacêutica contribui na 
adesão ao tratamento. Pode-se enfatizar também que a adesão ao tratamento correto vai ao encontro do uso racional de medicamentos, já que estes são peça chave na melhoria dos resultados da saúde pública aos cuidados de saúde (NGUYEN; ROUGHEAD, 2018).

Para a realização do uso racional de medicamentos é essencial que os países utilizem uma política nacional de medicamentos, a partir de ferramentas e estratégias interventivas para melhorias do serviço prestado. O medicamento, quando utilizado de forma adequada, é um recurso terapêutico essencial para as políticas de saúde (SILVA et al., 2017; HARDON; HODGKIN, 2004).

Conforme relatos de alguns pacientes no presente estudo, a farmacêutica utiliza recursos para facilitar a compreensão do tratamento. Este dado corrobora outra pesquisa, na qual se ressalta que dos 819 pacientes que se confundiram na utilização dos medicamentos, 712 (87\%) receberam orientação padronizada com cores e pictogramas, e $82 \%$ destes voltaram a usar seus medicamentos nos meses seguintes e seguiram a posologia correta, conforme a prescrição médica (MELO et al., 2017).

É importante ressaltar que, no presente estudo, a farmacêutica não foi identificada por alguns pacientes como profissional de saúde. Este dado pode estar relacionado com a comunicação não verbal expressada por muitos profissionais e, segundo a literatura, representa a grande maioria da comunicação diária com os pacientes, como por exemplo, a utilização do uso de jaleco. Esta comunicação não verbal pode resultar no respeito e na credibilidade, transmitindo a imagem de identidade ao profissional de saúde (MARTINS; MARTINS, 2011).

Portanto esse estudo mostrou a importância de ter o farmacêutico na UBS, pois ele é fundamental na organização da assistência na atenção básica. No entanto, na realidade de vários municípios do país, ainda se repete a ausência do profissional na maior parte das UBS (DOBLINSKI, 2011; SANTOS; ROSA; LEITE, 2017), embora seja exigida legalmente sua presença (CONSELHO FEDERAL DE FARMÁCIA, 1973). 


\section{CONCLUSÃO}

A profissão está evoluindo e acaba por exigir a diversificação do papel do farmacêutico, indo além do ato de dispensar medicamentos.

Observa-se que a presença da farmacêutica na UBS do município de Criciúma foi de fundamental importância para o alcance dos resultados positivos, principalmente em relação às intervenções, elaboradas na forma de encaminhamentos aos prescritores, em que houve aceitabilidade por grande parte dos mesmos $(72,72 \%)$.

Quanto ao percentual da orientação fornecida pela farmacêutica e sua contribuição para a adesão ao tratamento, obteve-se aproximadamente $92 \%$ das respostas positivas. Estes dados demonstram a importância do papel do farmacêutico profissional para um melhor resultado no tratamento, ao agir sempre com segurança, paciência e conhecimentos técnicos, garantindo à população cuidado e saúde de qualidade.

Outros estudos como este devem ser realizados para reafirmar a importância da presença do farmacêutico nas unidades de saúde do país.

\section{REFERÊNCIAS}

ANDRADE, F. C. D. et al. Life expectancy with and without cognitive impairment among Brazilian older adults. Archives of Gerontology and Geriatrics. ed. 58 (2014), p. 219-225. 2013.

ALVES, H. H. S. et al. Perspectiva sobre o entendimento do cuidado farmacêutico ao idoso em uma instituição filantrópica. Santa Maria, v. 43, n. 1, p. 140-147, jan./abr. 2017.

ANDRADE, M. A.; SILVA, M. V. S.; FREITAS, O. Pharmaceutical Assistance as a Strategy for the Rational Use of Medicine by the Elderly. Ciência e Saúde Coletiva. 2005. 
AQUINO, D. S. Por que o uso racional de medicamentos deve ser uma prioridade? Revista Ciência \& Saúde Coletiva, v. 13, n. 1, jan. 2008.

AZEVEDO, M. G. B. et al. Effectiveness of home pharmaceutical interventions in metabolic syndrome: a randomized controlled trial. Braz. J. Pharm. Sci. v. 53, n. 2, p. e16089-, jan. 2017.

BERNARDI, E. A. T. et al. Implantação da avaliação farmacêutica da prescrição médica e as ações de farmácia clínica em um hospital oncológico do sul do Brasil. Revista Espaço para a Saúde, v. 15, n. 2, jun. 2014.

BRASIL. Portaria n. 3.916, de 30 de outubro de 1998. Política Nacional de Medicamentos. Diário Oficial da União. 1998.

BRASIL. Portaria n. 834, de 14 de maio de 2013a. Redefine o comitê Nacional para promoção do uso racional de medicamentos no âmbito do Ministério da saúde. Diário Oficial da União. 2013.

BRASIL. Resolução n. 585, de 29 de agosto de 2013b. Regulamenta as atribuições clínicas do farmacêutico e dá outras providências. Diário Oficial da União. 2013.

BRASIL. Resolução n. 586, de 29 de agosto de 2013c. Regula a prescrição farmacêutica e dá outras providências. Diário Oficial da União. 2013.

BRASIL. Rev. Bras. Geriatr. Gerontol. Rio de Janeiro, 2015; v. 18, n. 1, p. 151-164.

CAVACO, A. M. et al. Self-medication and non-prescription drug counseling: Illustrating profession uncertainty within Turkish pharmacy practice. Research in Social and Administrative Pharmacy. v. 14, p. 718-726. 2018.

CONSELHO FEDERAL DE FARMÁCIA. Lei n. 5.991, de 17 de novembro de 1973. Dispõe sobre o Controle Sanitário do Comércio de Drogas, Medicamentos, Insumos Farmacêuticos e Correlatos, e dá outras providências. Diário Oficial da União. 1973. 
COSTA, C. M. F. N. et al. Use of medicines by patients of the primary health care of the Brazilian Unified Health System. Revista de Saúde Pública, v. 51, n. 2,22 set. 2017.

CURRAN, G. M. et al. Communication between pharmacists and primary care physicians in the midst of a U.S. opioid crisis. Research in Social and Administrative Pharmacy. Pii: S1551-7411 (18) 30302-4. doi: 10.1016.2018.

DAWALIBI, N. W. et al. Envelhecimento e qualidade de vida: análise da produção científica da Scielo. Estudos de Psicologia. Campinas-SP. v. 30, n. 3, p. 393-403, jul./set. 2013.

DOBLINSKI, P. M. F. A dispensação de medicamentos em Unidades Básicas de Saúde do município de Toledo-PR. Dissertação (Dissertação de mestrado). Universidade Federal de Santa Catarina. Florianópolis-SC, 2011.

ESCOREL, S. et al. O programa de saúde da família e a construção de um novo modelo para a atenção básica no Brasil. Revista Panamericana de Salud Pública, v. 21, n. 2, out. 2007.

GIOVANELLA, L.; MENDONA, MHM. Atenção Primária à Saúde: seletiva ou coordenadora dos cuidados? / Lígia Giovanella, Maria Helena Magalhães de Mendonça. Rio de Janeiro: Centro Brasileiro de Estudos da Saúde. 2012.

GOIS, A. C. D.; PERNAMBUCO, L.; LIMA, KC. Prevalence and Associated Factors With Voice Disorders in Brazilian Community-dwelling Older Adults. Journal of Voice. 2018.

GONÇALVES, F. C.; FARIA, C. C. C. O acesso aos serviços de saúde: uma análise na perspectiva do gênero. Revista Perquirere, v. 13, n. 1, p. 135-147, jul. 2016.

GOURLART, M. T. C. et al. Performance of a Brazilian population on the test of functional health literacy in adults. Revista de Saúde Pública. v. 43, n. 4, p. 631-8. 2009. 
HARDON, A.; HODGKIN, C.; FRESLE, D. How to investigate use of medicines by consumers. Switzerland: WHO/University of Amsterdam/ Royal Tropical Institute; 2004.

LEITE, S. N. et al. Assistência farmacêutica no Brasil: política, gestão e clínica. Editora da UFSC, v. 2, 2016.

LEVORATO, C. D. et al. Fatores associados à procura por serviços de saúde numa perspectiva relacional de gênero. Ciência e saúde coletiva [online]. 2014, v. 19, n. 4, p.1263-1274.

LOMBARDI, N. F. O serviço de cuidado farmacêutico na Atenção Primária à Saúde no município de Curitiba-PR. Dissertação. (Dissertação de Mestrado). Universidade Federal do Paraná. 2016.

MAEYAMA, M. A. et al. Sérgio Arouca: Relato de Experiência. Revista Brasileira de Educação Médica, v. 42, n. 1, jan. 2018.

MACHADO, L. F.; ALMEIDA, L. F. Avaliação do uso de omeprazol pelos pacientes do grupo de hipertensos do município de Inhaúma-MG. Faculdade Ciências da Vida - FCV. 2017.

MALTA, D. C. et al. The implantation of the Surveillance System for Noncommunicable Diseases in Brazil, 2003 to 2015: successes and challenges. Revista Brasileira de Epidemiologia. v. 20, n. 4, p. 661-675, out./dez. 2017.

MALTA, D. C. et al. Mortality due to noncommunicable diseases in Brazil, 1990 to 2015, according to estimates from the Global Burden of Disease study. São Paulo. Med Journal. v. 135, n. 3, p. 213-21. 2017.

MANSO, M. E. G.; BIFFI, E. C. A.; GERARDI, T. J. Prescrição inadequada de medicamentos a idosos portadores de doenças crônicas em um plano de saúde no município de São Paulo. Brasil. Rev. Bras. Geriatr Gerontol. Rio de Janeiro, v. 18, n. 1, p. 151-164. 2015.

MARAGNO, C. A. D.; LUIZ, P. P. V. Letramento em saúde e adesão ao tratamento medicamentoso: uma revisão da literatura. Revista Iniciação Científica. Criciúma, v. 14, n. 1, 2016. 
MARIM, N. et al. Assistência farmacêutica para gerentes municipais. OPAS/ OMS, 2003.

MARIN, M. J. S. et al. Caracterização do uso de medicamentos entre idosos de uma unidade do Programa Saúde da Família. Caderno de Saúde Pública, v. 24, n. 7, jul. 2008.

MARTIN, M. T.; FABER, M. T.; FABER, D. M. Patient satisfaction with the clinical pharmacist and prescribers during hepatitis $\mathrm{C}$ virus management. Journal Of Clinical Pharmacy And Therapeutics, v. 41, n. 6, 31 ago. 2016.

MARTINS, E. F.; MARTINS, E. F. O uniforme enquanto objeto sígnico na área da saúde. Verso e Reverso, v. 15, n. 59, maio-ago. 2011.

MELO, D. O.; CASTRO, L. L. C. D. A contribuição do farmacêutico para a promoção do acesso e uso racional de medicamentos essenciais no SUS. Ciência \& Saúde Coletiva, v. 22, n. 1, jan. 2017.

MELO, D. O. et al. Training of pharmacy technicians for dispensing drugs in Primary Health Care. Ciênc Saúde Coletiva, Rio de Janeiro, v. 22, n. 1, p. 261268, jan. 2017.

MINISTÉRIO DA SAÚDE. Política Nacional de Atenção Básica, 1. ed., 2012. MINISTÉRIO DA SAÚDE. Cartilha para a promoção do uso racional de medicamentos, 1. ed., 2015

MINISTÉRIO DA SAÚDE. Cuidado farmacêutico na atenção básica: Caderno n. 1: Serviços farmacêuticos na atenção básica à saúde, 1. ed., 2014. MINISTÉRIO DA SAÚDE. Atenção à Saúde da pessoa idosa e envelhecimento. Série Pactos pela Saúde. 2006, v. 12. Brasília. 2010.

NGUYEN, T. A.; ROUGHEAD, E. E. Strengths and weaknesses of pharmaceutical policy in relation to rational and responsible medicines use. Social and Administrative Aspects of Pharmacy in Low- and MiddleIncome Countries. p. 247-257. 2018. 
OLIVEIRA, M. M. et al. A saúde do homem em questão: busca por atendimento na atenção básica de saúde. Ciênc Saúde Coletiva, Rio de Janeiro, v. 20, n. 1, p. 273-278, jan. 2015.

ORGANIZAÇÃO MUNDIAL DA SAÚDE. Uso Racional de Medicamentos: fundamentação em condutas terapêuticas e nos macroprocessos da Assistência Farmacêutica. Brasília, dez. 2015.

PINTO, L. H. et al. O uso racional de medicamentos no brasil dentro da assistência farmacêutica brasileira e suas implicações no presente. Revista Eletrônica de Farmácia, v. 12, n. 1, maio 2015.

RODRIGUES, F. F. L. et al. Relação entre conhecimento, atitude, escolaridade e tempo de doença em indivíduos com diabetes mellitus. Acta Paul Enfermagem. 2; 25(2): 284-90. 2012.

ROZENFELD, S.; FONSECA, M. J. M.; ACURCIO, F. A. Drug utilization and polypharmacy among the elderly: a survey in Rio de Janeiro City, Brazil. Revista Panamericana de Salud Pública, v. 23, n. 1, jan. 2008.

SAMPAIO, H. A. C. et al. Letramento em saúde de diabéticos tipo 2: fatores associados e controle glicêmico. Ciência \& Saúde Coletiva, 20 (3): 865-874, 2015.

SANTOS, V. B.; ROSA, O. S.; LEITE, F. M. C. A importância do papel do farmacêutico na Atenção Básica. Rev Bras Pesq Saúde. Vitória, v. 19, n. 1, p. 39-43, jan./mar. 2017.

SCHENKEL, E. P.; MENGUE, S. S.; PETROVICK, P. R. Cuidados com os medicamentos. 5. ed. rev. Florianópolis: Ed. UFSC, 2012. 255p.

SCHMIDT, M. I. et al. Doenças crônicas não transmissíveis no Brasil: carga e desafios atuais. Saúde no Brasil: a série The Lancet. Rio de Janeiro: Fiocruz; 2011.p. 61-74.

SCHWEITZER, A. C. D. et al. Levantamento de dados acerca do uso racional de medicamentos pelos idosos que moram sozinhos na cidade de Fraiburgo - Santa Catarina. Ries, ISSN 2238-832X, Caçador, v. 7, n. 1, p. 220-232, 2018. 
SILVA, A. S. et al. Indicadores do uso de medicamentos na atenção primária de saúde: uma revisão sistemática. Rev Panam Salud Publica. 2017; n. 41, p. 132. doi: 10.26633/RPSP.2017.132.

SINITOX. Sistema Nacional de Informações Tóxico-Farmacológicas (Brasil). Fundação Oswaldo Cruz. Casos Registrados de Intoxicação Humana por Agente Tóxico e Zona de Ocorrência. Brasil, 2016.

TANAKA, O. Y. Primary health care evaluation: a new approach. Saúde e Sociedade, v. 20, n. 4, dez. 2011.

TONELI, M. J. F.; SOUZA, M. G. C.; MULLER, R. C. F. Masculinidades e práticas de saúde: 973 retratos da experiencia de pesquisa em Florianópolis/ SC. Physis Revista de Saúde Coletiva. Rio de Janeiro, v. 20, n. 3, p. 973-994, 2010.

VASCONCELOS, D. M. M. et al. Política Nacional de Medicamentos em retrospectiva: um balanço de (quase) 20 anos de implementação. Ciência \& Saúde Coletiva, v. 22, n. 8, ago. 2017.

VIANA, S. S. C.; ARANTES, T.; RIBEIRO, S. C. C. Intervention of the clinical pharmacist in an Intermediate Care Unit for elderly patients. Einstein. v. 15, n. 3, set. 2017 .

YEGENOGLU, S.; BAYDAR, T. Information and observations of Community pharmacists on geriatric patients: a qualitative study in ankara city. Turkish Journal of Geriatrics. 2011; 14 (4) 344-351.

WEISS, B. D. et al. Quick Assessment of Literacy in Primary Care: The Newest Vital Sign. Annals of Family medicine. v. 3, n. 6, nov./dez. 2005.

WIDAYATI, A. et al. Pharmacists' views on the development of asthma pharmaceutical care model in Indonesia: A needs analysis study. Research in Social and Administrative Pharmacy. 2017.

WORLD HEALTH ORGANIZATION. The rational use of drugs: report of the conference of experts. Nov. 1985. 